\section{Acetylation regulates subcellular localization of the Wnt signaling nuclear effector POP-1}

\author{
Frédérique Gay, ${ }^{1}$ Dominica Calvo, ${ }^{1,4}$ \\ Miao-Chia Lo, ${ }^{2,4}$ Julian Ceron, ${ }^{1}$ Morris Maduro, ${ }^{3}$ \\ Rueyling Lin, ${ }^{2}$ and Yang $\mathrm{Shi}^{1,5}$ \\ ${ }^{1}$ Department of Pathology, Harvard Medical School, Boston, \\ Massachusetts 02115, USA; ${ }^{2}$ Department of Molecular \\ Biology, University of Texas Southwestern Medical Center, \\ Dallas, Texas 75390-9148, USA; ${ }^{3}$ Department of Molecular, \\ Cellular, and Developmental Biology and Neuroscience \\ Research Institute, University of California, \\ Santa Barbara, California 93106, USA
}

Lymphoid enhancer factor/T-cell factor (LEF/TCF) are transcription factors that mediate the Wnt signaling pathway, and have crucial roles during embryonic development in various organisms. Here we report that acetylation enhances nuclear retention of POP-1, the Caenorhabditis elegans LEF/TCF homolog, through increasing nuclear import and blocking nuclear export. We identify three lysines that are acetylated in vivo, and demonstrate their essential requirement for proper nuclear localization and biological activity of POP-1 during C. elegans embryogenesis. The conservation of these lysines among other LEF/TCF family members suggests that acetylation may be an important, evolutionarily conserved mechanism regulating subcellular distribution of LEF/TCF factors.

Supplemental material is available at http://www.genesdev.org.

Received September 17, 2002; revised version accepted January 30, 2003.

Lymphoid enhancer factor/T-cell factor (LEF/TCF) transcription factors are conserved from Caenorhabditis elegans to humans, and are nuclear effectors of the Wnt signaling pathway that not only controls cell fate decisions and embryonic patterning during development but is also implicated in carcinogenesis (Van Noort and Clevers 2002). LEF/TCF factors all share a conserved high-mobility group (HMG) DNA-binding domain, and the ability to interact with Armadillo/ $\beta$-catenin, a key downstream component of the Wnt pathway (Eastman and Grosschedl 1999; Korswagen et al. 2000). In the absence of Wnt signal, LEF/TCF factors actively repress Wnt target gene expression (Eastman and Grosschedl 1999; Calvo et al. 2001). Activation of the Wnt pathway results in the stabilization and subsequent translocation

[Keywords: POP-1; LEF/TCF; Wnt; CBP-1; acetylation; C. elegans]

${ }^{4}$ These authors contributed equally to this work.

${ }^{5}$ Corresponding author.

E-MAIL yang shi@hms.harvard.edu; FAX (617) 432-1313.

Article and publication are at http://www.genesdev.org/cgi/doi/10.1101/ gad.1042403 of $\beta$-catenin into the nucleus, allowing transcriptional activation of the Wnt targets (Eastman and Grosschedl 1999). The C. elegans LEF/TCF homolog, POP-1, has been shown recently to function in such a canonical Wnt pathway regulating cell migration (Korswagen et al. 2000; Herman 2001). POP-1 also mediates the effects of a noncanonical Wnt pathway to control the polarity of EMS, the common precursor of endodermal (E) and mesodermal (MS) cells (Lin et al. 1995, 1998; Thorpe et al. 1997; Shin et al. 1999; Herman 2001).

During C. elegans early embryogenesis, the converging actions of Wnt and MAPK-related signaling pathways polarize the EMS blastomere, leading to the birth of two nonequivalent sister cells, the anterior MS and the posterior E cell, and setting the stage for the future differentiation of the mesoderm and endoderm tissues, respectively (Thorpe et al. 1997; Meneghini et al. 1999; Rocheleau et al. 1999; Shin et al. 1999). A mutation in the pop-1 gene results in the MS cell adopting the fate of its posterior sister $\mathrm{E}$ and producing endoderm (Lin et al. 1995, 1998). This phenotype indicates an essential role for POP-1 in cell fate specification, and suggests that POP-1 suppresses acquisition of the E fate in MS. Consistently, POP-1 has been shown to prevent the MS cell from adopting the endodermal fate through repressing transcription of the early endoderm-determining gene end-1 (Calvo et al. 2001). In contrast to the MS to E conversion observed in the pop-1 mutant background, mutations affecting Wnt/MAPK pathway components result in embryos that lack endoderm and overproduce mesoderm (Thorpe et al. 1997; Rocheleau et al. 1999; Meneghini et al. 1999|. Together with the observation that POP-1 nuclear level is higher in the unsignaled MS cell than in the Wnt/MAPK-targeted E cell (Lin et al. 1998), these data have led to the hypothesis that the Wnt/MAPK pathways down-regulate POP-1 activity in the posterior E cell, alleviating POP-1-dependent transcriptional repression and allowing endoderm differentiation. Although POP-1 down-regulation has been proposed to involve Wnt/MAPK-dependent phosphorylation and nucleocytoplasmic redistribution of POP-1 (Rocheleau et al. 1999; Maduro et al. 2002), the molecular mechanisms regulating its functions remain largely unknown. Here we report that acetylation regulates POP-1 subcellular localization and biological activity during C. elegans early embryogenesis.

\section{Results and Discussion}

$C B P / p 300$ proteins acetylate POP-1 in vitro and in vivo

As shown in Figure 1A, immunoprecipitates isolated from cells transfected with Flag-p300, but not empty Flag vector (cf. +p300 and -p300), acetylated GST-POP1 , but not GST alone, in vitro. Only the HMG-box DNAbinding domain (amino acids 188-281) and the region between the putative $\beta$-catenin-binding domain (amino acids 1-47) and the HMG-box, that is, amino acids 48187, are significantly acetylated by p300 (Fig. 1B). Because the $\beta$-catenin interacting domain (amino acids 1-47) alone does not interact with p300 in vitro (Supplementary Fig. 1), it might nonetheless be acetylated in the 
Figure 1. $\mathrm{CBP} / \mathrm{p} 300$ proteins acetylate POP-1. (A) Full-length POP-1 was acetylated by immunopurified human p300 $(+\mathrm{p} 300)$, but not immunoprecipitates recovered from empty vector-transfected cells $(-\mathrm{p} 300)$, in vitro. Arrowheads indicate the position of the GST and GST-POP-1 proteins. $(B)$ Truncated POP-1 proteins (arrowheads) were assayed for acetylation by human p300. (C) $\mathrm{K} 185$ is the main target of human p300 in vitro, as shown using GST-POP-1 fusion proteins carrying lysine $(\mathrm{K})$ to alanine $(\mathrm{A})$ substitutions of K185, K187, and/or K188 as substrates. (D) Recombinant human p300 and C. elegans CBP-1 acetylate fulllength POP-1 at K185 in vitro. (E) Acetylation of POP-1 in mammalian cells. POP-1 proteins were immunoprecipitated from COS-7 cells, and analyzed by Western blotting (WB) using the pan-AcK $(\alpha-A c K)$, the $\alpha$-AcK185, or the same $\alpha$-POP-1 antibody used for immunoprecipitation. (F) POP-1 is acetylated on K185, as well as K187, and/or K188 in vivo. GFP ::POP-1 fusion proteins were immunoprecipitated from $C$. elegans transgenic lines, and analyzed further as in $E$. $(G)$ CBP-1 acetylates POP-1 at K185 in vivo. Endogenous POP-1 was immunoprecipitated from wild-type or $c b p-1(R N A i)$ C. elegans embryos, and analyzed further by Western blotting (WB) using the $\alpha$-AcK185 antibody. Inhibition of CBP-1 expression was greater than $80 \%$ (data not shown). (H) Conservation of K185, K187, and K188 (indicated in bold) of POP-1 in the human and Drosophila POP-1 homologs.

context of the full-length POP-1 protein, as described for Drosophila TCF (Waltzer and Bienz 1998).

Using additional POP-1 deletion mutants, we next narrowed the acetylated region to amino acids 77-192, which contain four lysine (K) residues (K140, K185, $\mathrm{K} 187$, and K188). Analysis of point mutants ruled out the involvement of K140 (data not shown), suggesting that K185, K187, and/or K188, adjacent to the HMG-box DNA-binding domain (amino acids 188-281), are prime candidate sites for $\mathrm{CBP} / \mathrm{p} 300$-mediated acetylation. Consistently, simultaneous mutations of these three lysines to alanine (A) completely abolished acetylation (Fig. 1C, MutAAA). Substitution of K187 and K188 to A, while leaving K185 intact, did not significantly affect POP-1 acetylation level (Fig. 1C, MutKAA). In contrast, mutations leaving only K187 (MutAKA) or K188 (MutAAK) intact dramatically decreased acetylation (Fig. 1C). Therefore, although K187 and K188 are acetylated, K185 appears to be the main site of acetylation by p300 in vitro.

To further investigate K185 acetylation, we raised a polyclonal antibody against a synthetic peptide (POP-1 amino acids 179-191) with an acetyl group at K185. As shown in Figure 1D, recombinant human p300 and CBP1, the C. elegans homolog of mammalian CBP/p300 (Shi and Mello 1998), acetylated GST-POP-1 in vitro. Moreover, the $\alpha$-AcK185 antibody specifically recognized acetylated GST-POP-1 without cross-reacting with nonacetylated POP-1 (Fig. 1D). The specificity of this antibody was further demonstrated by analysis of wild-type, as well as various lysines to alanine or arginine (R) POP-1 point mutants in COS-7 cells (Fig. 1E). Immunopurified wild-type POP-1 was readily recognized by the pan-AcK antibody $(\alpha-\mathrm{AcK})$, demonstrating that POP- 1 is acetylated in mammalian cells. Simultaneous mutations of K185, K187, and K188 (Fig. 1E, MutAAA and MutRRR) drastically reduced POP-1 acetylation, suggesting that these residues are collectively accountable for the majority of POP-1 acetylation in vivo. The pan-AcK antibody also recognized all POP-1 mutants with simultaneous substitution of two of these three $\mathrm{K}$, indicating that $\mathrm{K} 185, \mathrm{~K} 187$, and $\mathrm{K} 188$ are all acetylated in COS-7 cells (Fig. 1E, MutKAA, MutAKA, and MutAAK). Significantly, the $\alpha$-AcK185 antibody recognized POP-1 only

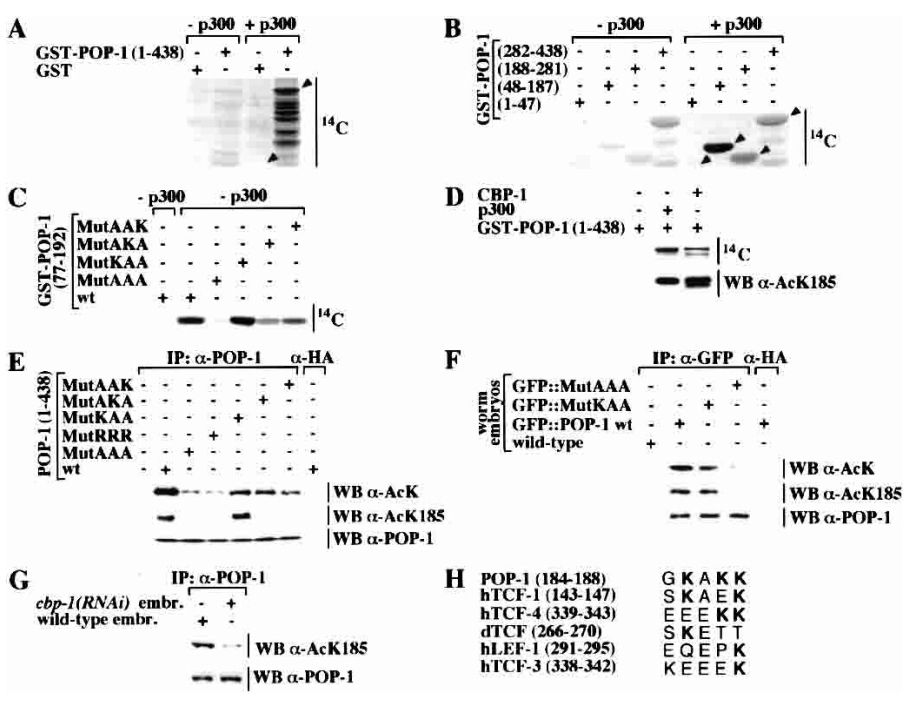

when K185 is intact (Fig. 1E, wild-type and MutKAA), thereby demonstrating the highly site-specific nature of this antibody.

We next investigated whether POP-1 is acetylated at $\mathrm{K} 185$, K187, and/or K188 in C. elegans embryos using worm transgenic lines expressing the wild-type POP-1, the MutAAA, or the MutKAA proteins fused to GFP. Wild-type GFP::POP-1 was shown previously to possess essentially the same biochemical and biological properties as endogenous POP-1 (Maduro et al. 2002). As shown in Figure 1F, GFP ::POP-1 was acetylated in worm embryos, and the overall level of acetylation was dramatically reduced by simultaneous mutations of K185, K187, and K188 (GFP::MutAAA). Mutation of K187 and K188 while leaving K185 intact also significantly decreased POP-1 acetylation, albeit to a lesser extent (Fig. 1F, GFP::MutKAA). Therefore K185, as well as K187 and/or $\mathrm{K} 188$, are acetylated in worm embryos. Endogenous POP-1 was also acetylated at K185 by CBP-1 in worm embryos as RNAi inhibition of CBP-1 expression drastically reduced K185 acetylation (Fig. 1G). Supporting this view, endogenous POP-1 and CBP-1 physically interact in worm embryos (Supplementary Fig. 1). Taken together, these results show that $\mathrm{CBP} / \mathrm{p} 300$ proteins interact with and acetylate POP-1, and identify K185, as well as $\mathrm{K} 187$ and/or K188, as the major acetylation sites in vivo. These findings may be relevant to other TCF/LEF members because, despite the low sequence homology within the region preceding the HMG-box, K185, K187, and/or K188 are found at similar locations in some mammalian and Drosophila LEF/TCFs (Fig. 1H).

\section{K185, K187, and K188 are required for proper nuclear localization and biological activity of POP-1 during C. elegans embryogenesis}

To determine whether these lysines are important for POP-1 function in vivo, we performed genetic rescue assays in the pop-1(zu189) mutant. This maternal-effect lethal mutation results in homozygous mothers laying embryos in which the MS cell adopts the fate of its posterior sister cell $\mathrm{E}$, producing endoderm instead of mesoderm (Lin et al. 1995). A transgene encoding GFP::POP-1 under the control of an EMS-specific pro- 
$\mathbf{A}$

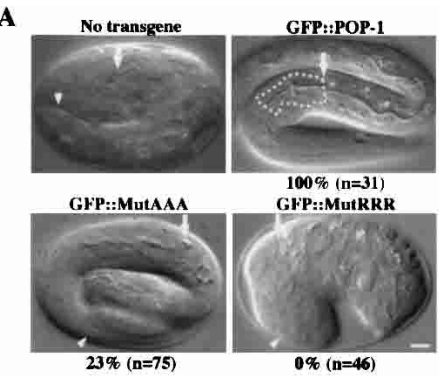

B

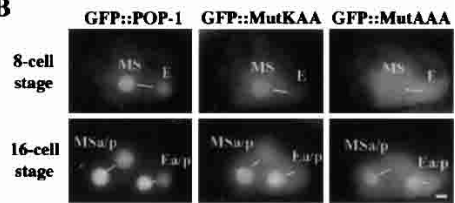

Figure 2. Activity and subcellular distribution of POP-1 mutants in C. elegans embryos. (A) Light micrographs of pop-1(zu189) mutant embryos carrying a med-1::gfp::pop-1 transgene encoding either the wild-type POP-1, or the MutAAA and MutRRR mutants. Because of the lack of posterior MS-derived pharynx (delimited by a broken line when present), pop-1(zu189) mutant embryos have a pharynx much smaller than wild-type (not shown) or pop-1 mutant embryos in which the pharyngeal defects are rescued by ectopic expression of wild-type POP-1. The anterior tip of the pharynx is indicated by a white arrowhead, and its posterior tip is indicated by a white arrow. For each transgene, the number of embryos scored and the percentage of embryos in which the posterior pharynx defects were successfully rescued are indicated. $(B)$ Fluorescence micrographs of 8- and 16-cell stage embryos expressing the wild-type POP-1, the MutKAA or the MutAAA mutants. The MS and E cells, and their descendants born from anterior/posterior $(\mathrm{a} / \mathrm{p})$ divisions are indicated. Anterior is to the left, and dorsal is up. Bar, $5 \mu \mathrm{m}$.

moter, med-1, has been shown recently to rescue the mispecification of the MS blastomere in pop-1(zu189) embryos (Maduro et al. 2002). We observed that $100 \%$ of the embryos carrying the med-1::gfp::pop-1 transgene produced MS-derived pharynx (Fig. 2A, GFP::POP-1). In contrast, no rescue was observed with the MutRRR mutant (Fig. 2A, 0\%). The rescuing activity of the MutAAA mutant was also significantly compromised (Fig. 2A, $23 \%$ ). Mutations of two out of these three lysines (MutKAA) only improved the rescuing ability to $67 \%$ ( $\mathrm{n}=61$; data not shown), suggesting that, in addition to $\mathrm{K} 185$, K187, and/or K188, are important for POP-1 biological function in vivo.

Analysis of its subcellular distribution in live embryos showed that GFP::POP-1 recapitulated the endogenous POP-1 expression pattern, that is, high nuclear level in the anterior MS cell, and low in the posterior sister E cell, and was predominantly nuclear in both MS and E cells at all developmental stages examined (Fig. 2B; data not shown). In contrast, we observed mostly cytoplasmic GFP signal in both MS and E in 8-cell stage embryos expressing the GFP::MutAAA mutant (Fig. 2B). At the 16-cell stage, when MS and E have divided, some nuclear signal became detectable but the cytoplasmic level of GFP:: MutAAA remained high (Fig. 2B). The asymmetry of POP-1 nuclear level between MS and E, as well as their a/p descendants, however, was not affected by mutation of the three lysines (Fig. 2B). No significant differences in subcellular localization were observed between the MutAAA and MutRRR mutants (data not shown), suggesting that their differential rescuing ability might reflect different effects of these mutations on other func- tions of POP-1. In this regard, the residual activity of the MutAAA protein might be attributable to alanines partially mimicking acetylated lysines. Finally, a significant fraction of the MutKAA protein was also mislocalized, albeit to a lesser extent than the MutAAA mutant (Fig. 2B). Taken together, these observations demonstrate that $\mathrm{K} 185$, as well as $\mathrm{K} 187$ and/or K188, are important for the maintenance of a high steady-state level of nuclear POP-1 and for its biological activity during embryogenesis, but are not required for POP-1 nuclear asymmetry.

\section{Mutation of K185, K187, and K188 impairs nuclear import and increases nuclear export of POP-1}

To gain insight into the mechanism underlying the mislocalization of the POP-1 acetylation-defective mutants, we analyzed their subcellular distribution in mammalian cells. Wild-type POP-1 was localized predominantly in the nucleus in half of the transfected cells, but was either equally distributed in the nuclear and the cytoplasmic compartments, or mainly cytoplasmic, in the other half of the cells (Fig. 3A). In contrast, the MutAAA and MutRRR mutants were predominantly cytoplasmic in almost all transfected cells (Fig. 3A; data not shown). Quantification of these results are summarized in Supplementary Table 1. Similarly, all three double mutants (MutKAA, MutAKA, and MutAAK) were mainly cytoplasmic, although to a slightly lesser extent (data not shown). Our cell culture results are consistent with the mislocalization of the POP-1 mutants observed in eight-cell stage worm embryos. The discrepancies between the complete cytoplasmic localization of the MutAAA mutant in COS-7 cells and its partial nuclear accumulation in 16-cell stage embryos might reflect developmental regulation of POP-1 that is absent in cell culture. Regardless, in both systems, mutations of K185, K187, and/or K188 impair nuclear localization of POP-1.

The failure of the POP-1 mutants to localize in the nucleus could be caused by a defect in nuclear import, and/or an increased export. To address this issue, we asked whether POP-1 is regulated by a nuclear export

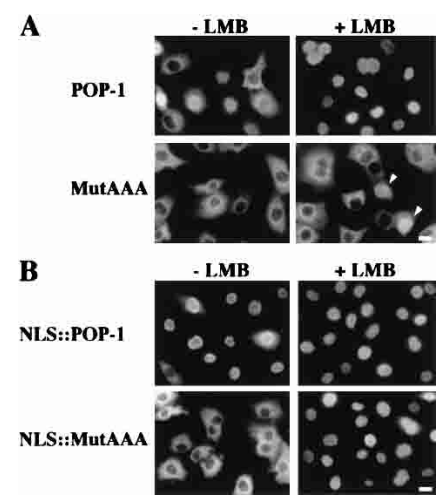

Figure 3. The MutAAA mutant is defective for nuclear import and displays enhanced nuclear export. $(A)$ COS-7 cells expressing the wild-type POP-1 or the MutAAA protein were treated or not with Leptomycin B (LMB). Arrowheads indicate cells in which the mutant protein is predominantly nuclear. $(B)$ Subcellular distribution of the wild-type POP-1 or the MutAAA mutant fused to the SV-40 NLS in COS-7 cells treated as in $A$. In both panels, the cells were stained with the monoclonal $\alpha$-POP-1 antibody. Bar, $20 \mu \mathrm{m}$. 
mechanism. Transfected COS-7 cells were treated with Leptomycin (LMB; Kudo et al. 1998), an inhibitor of the CRM-1-mediated nuclear export pathway (Fornerod et al. 1997). Whereas wild-type POP-1 was predominantly nuclear in only half of the cells, nearly $100 \%$ of the cells displayed a strict nuclear localization of POP-1 on LMB treatment (Fig. 3A; Supplementary Table 1), suggesting that POP-1 is regulated by nuclear export in mammalian cells. Although the MutAAA mutant became nuclear in a significant fraction of the transfected cells (indicated by arrowheads in Fig. 3A; N $>C=16 \%$ in Supplementary Table 1) when export was blocked, the majority of the mutant protein still remained cytoplasmic after LMB treatment. Similar results were obtained with the MutRRR mutant (Supplementary Table 1), showing that although the MutAAA and MutRRR proteins retain residual ability to enter the nucleus, they are by and large defective for nuclear entry.

We next analyzed the subcellular distribution of the wild-type and mutant POP-1 proteins fused to the SV-40 nuclear localization signal (NLS). In the absence of LMB, addition of the SV40 NLS increased the nuclear localization of wild-type POP-1 in COS-7 cells (Fig. 3B; Supplementary Table 1). As expected, NLS::POP-1 was nuclear in almost all transfected cells when nuclear export was blocked by LMB. Significantly, the NLS::MutAAA and NLS::MutRRR proteins became predominantly nuclear in most of the cells after LMB treatment, whereas they remained cytoplasmic in the absence of LMB (Fig. 3B; Supplementary Table 1). Collectively, these findings demonstrate that the mislocalization of the POP-1 mutants in mammalian cells is the result of both a decrease in nuclear entry and an enhancement in nuclear export. Significantly, addition of the SV-40 NLS also failed to promote nuclear localization of the MutAAA protein in worm embryos (data not shown), suggesting that POP-1 subcellular localization in worm embryos is regulated by a dual mechanism similar to that identified in mammalian cells.

\section{POP-1 subcellular distribution is regulated by a dynamic balance between acetylation and deacetylation}

We next investigated the functional significance of $\mathrm{K} 185$, K187, and K188 acetylation by analyzing the effect of the class I histone deacetylase inhibitor Trichostatin A (TSA; Taunton et al. 1996) on POP-1 subcellular localization (Fig. 4A; data not shown). Whereas TSA enhanced POP-1 nuclear localization $(\mathrm{N}>\mathrm{C}$ increased from $45.6 \%$ to $81.6 \%$ ), it had no significant effect on the subcellular distribution of the MutAAA and MutRRR mutants (Fig. 4A; data not shown). This strongly suggests that K185, K187, and K188 are required for TSAinduced nuclear localization of POP-1. Significantly, the effect of TSA on POP-1 subcellular distribution was correlated with a strong increase in the overall acetylation level of POP-1, as well as an enhancement of K185 acetylation (Fig. 4B). These data show that POP-1 subcellular localization is dynamically regulated by the antagonistic activities of acetylases and deacetylases, and provide a strong link between acetylation and the function of $\mathrm{K} 185, \mathrm{~K} 187$, and K188.

Our findings predict that nuclear POP-1 should be more acetylated than its cytoplasmic counterpart. As shown in Figure 4C, although the amounts of POP-1 im-
$\mathbf{A}$

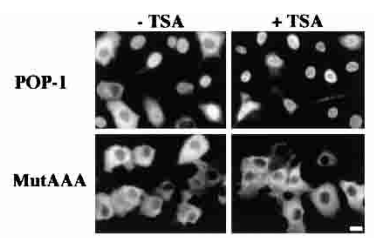

B

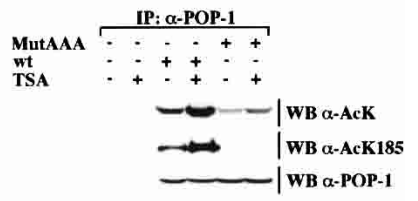

C

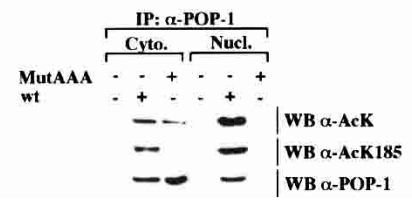

Figure 4. Acetylation at K185, K187, and/or K188 is required for POP-1 nuclear retention. (A) COS-7 cells expressing the wild-type POP-1 or the MutAAA mutant were cultured in the absence or presence of Trichostatin A (TSA), and stained with the monoclonal $\alpha$-POP-1 antibody. Bar, $20 \mu \mathrm{m}$. (B) The wild-type and mutant POP-1 proteins were immunoprecipitated from TSA-treated or untreated COS-7 cells, and analyzed by Western blotting (WB) using the panAcK $(\alpha-A c K)$, the $\alpha$-AcK 185 , or the same $\alpha$-POP-1 antibody used for immunoprecipitation. $(C)$ The indicated POP-1 proteins immunoprecipitated from COS-7 cytoplasmic and nuclear extracts were analyzed as in $B$.

munoprecipitated from both cellular fractions were comparable, the overall acetylation level of the nuclear POP-1 was significantly higher than that of the cytoplasmic POP-1, as was acetylation at K185 (Fig. 4C). In contrast, the MutAAA and MutRRR proteins were only detected in the cytoplasm, and were weakly acetylated (Fig. $4 \mathrm{C}$; data not shown). These results demonstrate that the pool of acetylated POP-1 is greatly enriched in the nuclear compartment, and provide a strong correlation between POP-1 acetylation and its nuclear retention.

The Wnt/MAPK pathway promotes POP-1 cytoplasmic redistribution by overriding the effect of acetylation

Consistent with the recent finding that POP-1 nuclear asymmetry results from a Wnt/MAPK-dependent nucleocytoplasmic redistribution of the protein (Maduro et al. 2002), the Lit-1 kinase and the $\beta$-catenin Wrm-1 have been shown to phosphorylate POP-1 and trigger its cytoplasmic relocalization in mammalian cells (Rocheleau et al. 1999). We used the same experimental approach to determine whether phosphorylation-induced cytoplasmic relocalization of POP-1 is mediated through a Wnt/ MAPK-dependent inhibition of its acetylation. As reported previously (Rocheleau et al. 1999), POP-1 became phosphorylated and accumulated in the cytoplasm of most of the transfected cells upon coexpression of Lit-1 and Wrm-1, but not of Lit-1 alone (data not shown). Phosphorylation of POP-1, however, affected neither its overall level of acetylation, nor its acetylation at K185 (Fig. 5). The fact that phosphorylation overrides the acetylation-induced nuclear retention of POP-1 without affecting its acetylation level per se suggests that phosphorylation and acetylation are independent regulatory events controlling the subcellular localization of POP-1. 


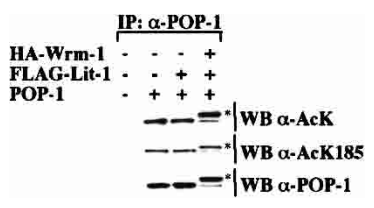

Figure 5. Phosphorylation of POP-1 does not affect its acetylation per se. Wild-type POP-1 immunoprecipitated from COS-7 cotransfected or not with Flag-tagged Lit-1 and HA-tagged Wrm-1 was analyzed by Western blotting (WB) using the pan-AcK antibody $(\alpha-\mathrm{AcK})$, the $\alpha$-AcK185 antibody, or the same $\alpha$-POP- 1 antibody used for immunoprecipitation. Phosphorylated POP-1 is indicated by an asterisk.

\section{Conclusions}

Although histones are by far the best characterized substrates for acetyltransferases, several of these enzymes have been shown recently to acetylate sequence-specific transcription factors, thereby modulating their DNAbinding activity, their ability to interact with cofactors and to regulate transcription, but also their nuclear retention (Soutoglou et al. 2000; Sterner and Berger 2000; Chen et al. 2001; Madison et al. 2002). Our results strongly suggest that acetylation and deacetylation regulate POP-1 subcellular localization. We identified K185, $\mathrm{K} 187$, and K188 as acetylation sites, and demonstrated their essential requirement for POP-1 nuclear retention and biological activity during C. elegans embryogenesis. Furthermore, we provided the first demonstration that a LEF/TCF factor is regulated by nuclear export, and showed that the defects of the POP-1 acetylation-defective mutants include a reduction in nuclear import and an enhancement of nuclear export. Significantly, the lysine residues identified as the acetylation sites are conserved among some LEF/TCF factors in other organisms, suggesting a possible evolutionary conservation of this regulatory mechanism.

In C. elegans, endoderm differentiation requires downregulation of POP-1 nuclear level and activity in the founder E cell, relieving its transcriptional repressor activity (Thorpe et al. 1997; Meneghini et al. 1999; Rocheleau et al. 1999; Shin et al. 1999; Calvo et al. 2001; Maduro et al. 2002). This critical event is controlled by the converging action of Wnt and MAPK signaling pathways (Rocheleau et al. 1999; Shin et al. 1999). Downregulation of POP-1 activity in E was shown recently to involve Wnt/MAPK-dependent alterations of its nucleocytoplasmic distribution and DNA-binding properties in C. elegans embryos, the former consisting of relocalization of POP-1 from the nucleus to the cytoplasm without significant degradation in either intracellular compartment (Maduro et al. 2002). These findings highlight the crucial role for subcellular localization control in the regulation of POP-1 biological activity in vivo and, together with our data, predict a potential functional antagonism between acetylation and Wnt/MAPK-dependent phosphorylation in this process. We showed that mutations of K185, K187, and K188 affect POP-1 subcellular localization without disrupting the anterior-posterior nuclear asymmetry. Therefore, POP-1 nuclear retention controlled by acetylation at these lysines is unlikely to be achieved by preventing Wnt/MAPK-induced POP-1 phosphorylation. Conversely, Wnt/MAPK pathways decrease POP-1 nuclear level without affecting its acetylation level, suggesting that phosphorylation can effectively counteract the effect of acetylation, perhaps by facilitating POP-1 interaction with the nuclear export machinery. The dynamic balance of these antagonistic activities in unsignaled versus Wnt-signaled cells is likely to have a critical role in the regulation of POP-1 nuclear retention and biological activity during $C$. elegans embryogenesis.

\section{Materials and methods}

Site-directed mutagenesis

All point mutations were generated using the QuikChange Site-Directed Mutagenesis Kit (Stratagene), and were verified by sequencing.

Generation of fusion proteins and in vitro acetylation assay GST fusion protein production and purification were performed as described (Gay et al. 2002), as were the in vitro acetylation reactions (Gu and Roeder 1997).

Preparation of protein extracts from worm embryos and mammalian cells

Total, nuclear, and cytoplasmic protein extracts were prepared as described (Calvo et al. 2001; Gay et al. 2002; Whetstine et al. 2002).

Immunoprecipitation and Western blotting

Immunoprecipitation and Western blotting were performed as described (Calvo et al. 2001; Gay et al. 2002). Flag-tagged human p300 was immunoprecipitated from HeLa cells using the $\alpha$-Flag antibody (Sigma). Wildtype and mutant POP-1 proteins were immunoprecipitated from worm embryos or transfected COS-7 cells using either the $\alpha$-POP-1 (Lin et al. 1995 ) or the $\alpha$-GFP (Santa Cruz) polyclonal antibodies, and were analyzed by Western Blotting using the pan-AcK ( $\alpha$-AcK, New England Biolabs), $\alpha$-AcK185, or $\alpha$-POP-1 antibodies.

Transient transfection and in situ immunofluorescence

COS-7 and HeLa cells were transfected as described (Gay et al. 2002). Trichostatin A $(1 \mu \mathrm{M})$ and Leptomycin B $(2 \mathrm{ng} / \mathrm{mL})$ were added to the medium for the last 24 and $4 \mathrm{~h}$ of culture, respectively. Cells were harvested $48 \mathrm{~h}$ after transfection and stained as described (Sui et al. 2002), using the mouse monoclonal $\alpha$-POP-1 antibody (Lin et al. 1998).

Generation of the $\alpha$-AcK185 antibody

A synthetic peptide spanning amino acids 179-191 of POP-1, in which K185 was preacetylated, was used to generate the $\alpha$-AcK185 rabbit polyclonal antibody (Covance).

RNA-mediated interference by feeding RNAi was performed as described previously (Timmons et al. 2001).

Generation of GFP::POP-1 constructs and transgenic C.

elegans animals

The wild-type POP-1 coding sequence was replaced by the MutAAA and MutRRR ones in the pMM414 vector, which allows expression of GFP::POP-1 under the control of the med-1 promoter and 3'UTR (Maduro et al. 2002). Transgenic animals were generated as described (Mello et al. 1991) using the unc-119 rescuing plasmid pPD\#MM016B as a transgenic marker into the unc-119 (ed4) strain.

Assay for POP-1 activity and localization in worm embryos Male transgenic animals were mated to homozygous pop-1(zu189) hermaphrodites, from which young embryos were collected 24 h later. GFPpositive embryos were allowed to develop for an additional $16 \mathrm{~h}$ (15 C) and scored for the development of the MS-derived pharynx using Nomarski optics. GFP fluorescence imaging was performed as described (Rogers et al. 2002). Images of live embryos were collected from single focal planes in grayscale at 30-sec intervals for at least three cell cycles, and images from selected time points are shown in Figure 2B.

\section{Acknowledgments}

We thank Gilles Salbert, Keith Blackwell, El Bachir Affar, Anjana Rao, and $\mathrm{Xi} \mathrm{He}$ for critical reading of the manuscript and helpful comments. We are grateful to Melanie Reuben and Pascale Dufourcq for technical 
assistance; and thank Joel Rothman, Minovu Yoshida, and Tae Ho Shin for providing us with reagents and plasmids. F.G. was supported by a fellowship from the Lalor Foundation, and from the Taplin Funds for Discovery. This work was supported by grants from the National Institutes of Health (GM58012, GM53874) to Y.S.

The publication costs of this article were defrayed in part by payment of page charges. This article must therefore be hereby marked "advertisement" in accordance with 18 USC section 1734 solely to indicate this fact.

\section{References}

Calvo, D., Victor, M., Gay, F., Sui, G., Luke, M.P., Dufourcq, P., Wen, G., Maduro, M., Rothman, J., and Shi, Y. 2001. POP-1 repressor complex restricts inappropriate cell type-specific gene transcription during Caenorhabditis elegans embryogenesis. EMBO J. 20: 7197-7208.

Chen, L.F., Fischle, W., Verdin, E., and Greene, W.C. 2001. Duration of nuclear NF-кB action regulated by reversible acetylation. Science 293: $1653-1657$.

Eastman, Q. and Grosschedl, R. 1999. Regulation of LEF-1/TCF transcription factors by Wnt and other signals. Curr. Opin. Cell. Biol. 11: 233-240.

Fornerod, M., Ohno, M., Yoshida, M., and Mattaj, I. 1997. CRM1 is an export receptor for leucine-rich nuclear export signals. Cell 90: 10511060.

Gay, F., Barath, P., Desbois-Le Peron, C., Métivier, R., Le Guevel, R. Birse, D., and Salbert G. 2002. Multiple phosphorylation events control chicken ovalbumin upstream promoter transcription factor I orphan nuclear receptor activity. Mol. Endocrinol. 16: 1332-1351.

Gu, W. and Roeder, R.G. 1997. Activation of p53 sequence-specific DNA binding by acetylation of the p53 C-terminal domain. Cell 90: 595606.

Herman, M. 2001. C. elegans POP-1/TCF functions in a canonical Wnt pathway that controls cell migration and in a noncanonical Wnt pathway that controls cell polarity. Development 128: 581-590.

Korswagen, H.C., Herman, M.A., and Clevers, H.C. 2000. Distinct $\beta$-catenins mediate adhesion and signalling functions in C. elegans. Nature 406: 527-532.

Kudo, N., Wolff, B., Sekimoto, T., Schreiner, E.P., Yoneda, Y., Yanagida, M., Horinouchi, S., and Yoshida, M. 1998. Leptomycin B inhibition of signal-mediated nuclear export by direct binding to CRM1. Exp. Cell Res. 242: 540-547.

Lin, R., Thompson, S., and Priess, J.R. 1995. pop-1 encodes an HMG box protein required for the specification of a mesoderm precursor in early C. elegans embryos. Cell 83: 599-609.

Lin, R., Hill, R.J., and Priess, J.R. 1998. POP-1 and anterior-posterior fate decisions in C. elegans embryos. Cell 92: 229-239.

Madison, D.L., Yaciuk, P., Kwok, R.P., and Lundblad, J.R. 2002. Acetylation of the adenovirus-transforming protein E1A determines nuclear localization by disrupting association with importin- $\alpha$. J. Biol. Chem. 277: 38755-38763.

Maduro, M.F., Lin, R., and Rothman, J.H. 2002. Dynamics of a developmental switch: Recursive intracellular and intranuclear redistribution of Caenorhabditis elegans POP-1 parallels Wnt-inhibited transcriptional repression. Dev. Biol. 248: 128-142.

Mello, C.C., Kramer, J.M., Stinchcomb, D., and Ambros, V. 1991. Efficient gene transfer in C. elegans: extrachromosomal maintenance and integration of transforming sequences. EMBO J. 10: 3959-3970.

Meneghini, M.D., Ishitani, T., Carter, J.C., Hisamoto, N., NinomiyaTsuji, J., Thorpe, C.J., Hamill, D.R., Matsumoto, K., and Bowerman, B. 1999. MAP kinase and Wnt pathways converge to downregulate and HMG-domain repressor in Caenorhabditis elegans. Nature 399: 793-797.

Rocheleau, C.E., Yasuda, J., Shin, T.H., Lin, R., Sawa, H., Okano, H., Priess, J.R., Davis, R.J., and Mello, C.C. 1999. WRM-1 activates the LIT-1 protein kinase to transduce anterior/posterior polarity signals in C. elegans. Cell 97: 717-726.

Rogers, E., Bishop, J.D., Waddle, J.A., Schumacher, J.M., and Lin, R. 2002. The aurora kinase AIR-2 functions in the release of chromosome cohesion in Caenorhabditis elegans. J. Cell. Biol. 157: 219-229.

Shi, Y. and Mello, C.C. 1998. A CBP/p300 homolog specifies multiple differentiation pathways in Caenorhabditis elegans. Genes \& Dev. 12: $943-955$.
Shin, T.H., Yasuda, J., Rocheleau, C.E., Lin, R., Soto, M., Bei, Y., Davis, R.J., and Mello, C.C. 1999. MOM-4, a MAP kinase kinase kinaserelated protein, activates WRM-1/LIT-1 kinase to transduce anterior/ posterior polarity signals in C. elegans. Mol. Cell 4: 275-280.

Soutoglou, E., Katrakili, N., and Talianidis, I. 2000. Acetylation regulates transcription factor activity at multiple levels. Mol. Cell. 5: 745-751.

Sterner, D.E. and Berger, S.L. 2000. Acetylation of histones and transcription-related factors. Mol. Cell. Biol. Rev. 64: 435-459.

Sui, G.C., Soohoo, C., Affar, E.B., Gay, F., Shi, Y., Forrester, W.C., and Shi, Y. 2002. A DNA vector-based RNAi technology to suppress gene expression in mammalian cells. Proc. Nat1. Acad. Sci. 99: 5515-5520.

Taunton, J., Hassig, C.A., and Schreiber, S.L. 1996. A mammalian histone deacetylase related to the yeast transcriptional regulator Rpd3p. Science 272: 408-411.

Thorpe, C.J., Schlesinger, A., Carter, J.C., and Bowerman, B. 1997. Wnt signaling polarizes an early C. elegans blastomere to distinguish endoderm and mesoderm. Cell 90: 695-705.

Timmons, L., Court, D.L., and Fire, A. 2001. Ingestion of bacterially expressed dsRNAs can produce specific and potent genetic interference in Caenorhabditis elegans. Gene 263: 103-112.

Van Noort, M. and Clevers, H. 2002. TCF transcription factors, mediators of Wnt-signaling in development and cancer. Dev. Biol. 244: 1-8.

Waltzer, L. and Bienz, M. 1998. Drosophila CBP represses the transcription factor TCF to antagonize Wingless signalling. Nature 395: 521525

Whetstine, J.R., Witt, T.L., and Matherly, L.H. 2002. The human reduced folate carrier gene is regulated by the AP2 and sp1 transcription factor families and a functional 61-base pair polymorphism. J. Biol. Chem. 277: 43873-43880 


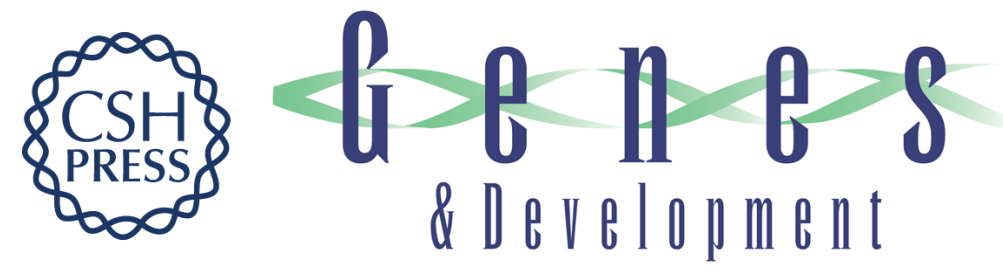

\section{Acetylation regulates subcellular localization of the Wnt signaling nuclear effector POP-1}

Frédérique Gay, Dominica Calvo, Miao-Chia Lo, et al.

Genes Dev. 2003, 17:

Access the most recent version at doi:10.1101/gad.1042403

\section{Supplemental http://genesdev.cshlp.org/content/suppl/2003/03/31/17.6.717.DC1 Material}

References This article cites 28 articles, 9 of which can be accessed free at: http://genesdev.cshlp.org/content/17/6/717.full.html\#ref-list-1

\section{License}

Email Alerting

Receive free email alerts when new articles cite this article - sign up in the box at the top Service

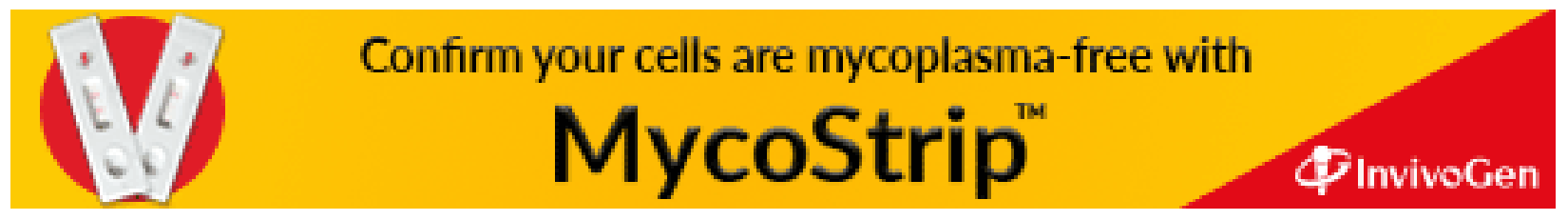

